\title{
Communication
}

\section{Aspects pratiques en élevage d'aulacodes (Thryonomys swinderianus). V. Croissance et usure normales des incisives}

\author{
G.A. Mensah ${ }^{1}$
}

\author{
A.Brönnemann ${ }^{1}$ \\ C.H. Stier ${ }^{1}$ \\ C.F. Gall ${ }^{1}$
}

MENSAH (G.A.), BRÖNNEMANN (A.), STIER (C.H.), GALL (C.F.) Aspects pratiques en élevage d'aulacodes (Thryonomys su'inderianus). V. Croissance et usure normales des incisives. Reviue Élev. Méd. vét. Pays trop., 1992, 45 (2) : 175-178

Chez les aulacodes d'élevage, on peut observer une usure mauvaise et insuffisante des incisives. Pour comprendre l'origine de ce phénomène, la croissance et l'usure de ces dernières ont été étudiées chez des aulacodes âgés de 4 à 12 mois. Les incisives poussent de $0,039-0,055 \mathrm{~mm} / \mathrm{j}$ our et leur usure est de 0,033-0,040 mm/jour. Il en résulte un surcroît de 0,006 $0,015 \mathrm{~mm} /$ jour qui toutefois n'a engendré aucun problème dentaire. Mots clés : Aulacode - Thryonomys su'inderianus - Élevage - Dent - Usure Bénin.

\section{Introduction}

La formule dentaire définitive de l'aulacode (Thryonomys swinderianus) adulte comprend quatre incisives et seize molaires, tandis que les jeunes aulacodes naissent avec deux paires d'incisives seulement. Les incisives sont courbées et font saillie hors de la bouche. Les incisives supérieures sont taillées en biseau chez les jeunes aulacodes, alors que chez l'aulacode sub-adulte et l'adulte les incisives supérieures ont à leur base une encoche d'environ $2 \mathrm{~mm}$ de profondeur. Dans cette encoche viennent se loger les extrémités des incisives inférieures lorsque l'animal s'alimente (1). Les incisives supérieures, jaune-orangé et convexes, portent sur la face extérieure trois sillons longitudinaux, alors que les inférieures jaunes sont lisses sur la face extérieure (fig. 1). Leur croissance continue compense l'abrasion des extrémités libres (1).

L'usure anormale des incisives des aulacodes constitue un problème d'élevage majeur à la station du Projet bénino-allemand d'aulacodiculture (PBAA) à Godomey (Bénin). Elle cause de lourdes pertes dans le cheptel (1, $2,3)$. II était donc impératif de résoudre ce problème avant qu'il ne devienne un facteur limitant pour la production de viande.

\footnotetext{
1. Institut de production animale des pays tropicaux et subtropicaux, Université de Hohenheim (480), Garbenstraße 17, D-7000 Stuttgart 70 , Allemagne.
}

Reçu le 5.8.1991, accepté le 26.6.1992

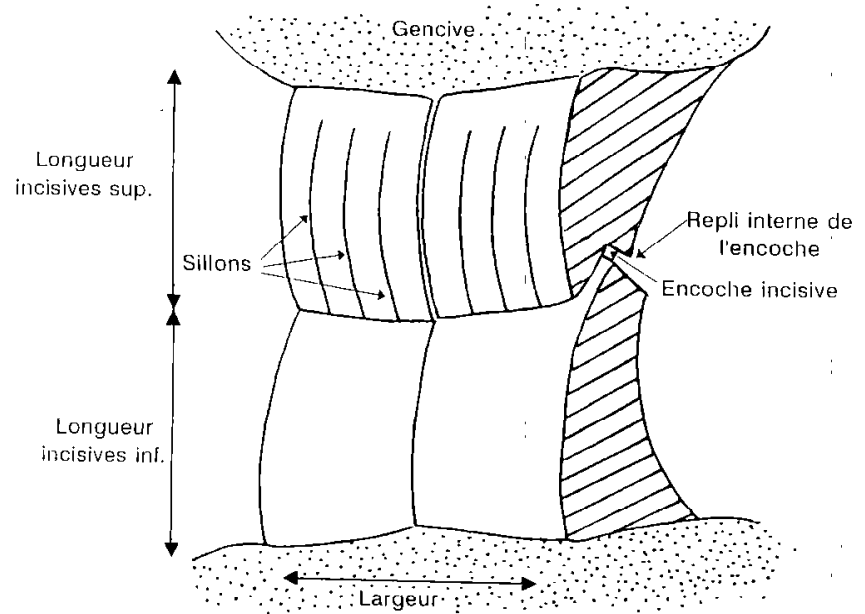

Figure I : Incisives vues des trois-quart avec indication des mesures prises.

ADJANOHOUN (1) a relevé trois principaux types d'anomalies dues à l'usure anormale des incisives de l'aulacode, et résultant probablement de l'absence de certains éléments permettant à l'animal à l'état sauvage d'entretenir ses dents :

- une croissance trop rapide des incisives supérieures par rapport aux inférieures (photo 1);

- une croissance trop rapide des incisives inférieures par rapport aux supérieures ;
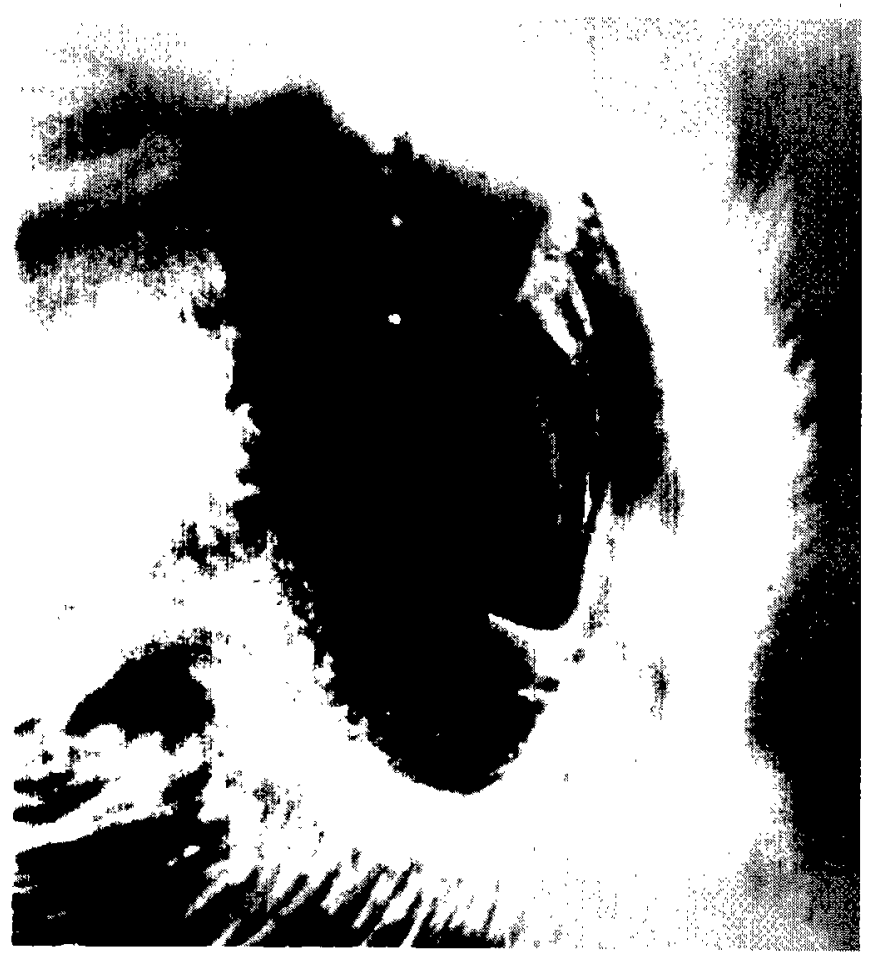

Photo I : Incisives supérieures plus longues que les inférieures. 
Communication

- une mauvaise usure du repli interne de l'encoche de l'incisive supérieure : malgré une bonne longueur, un parfait affrontement et une bonne usure apparente des incisives, on observe parfois un repli qui donne une fine lame qui finit par venir s'implanter dans les palais (photos 2, 3 et 4).

Afin de mieux appréhender ces problèmes dentaires, la croissance et l'usure normales des incisives de l'aulacode ont été étudiées ici.

\section{Matériel et méthode}

Les données ont été collectées dans la station d'élevage du PBAA. Les aulacodes étaient nourris ad libitum avec une ration de base de fourrages verts (Paspalum vaginatum et Pennisetum purpureum) et un complément des granulés. Ils étaient élevés en cage individuelle. Les mesures ont été prises sur les animaux sous anesthésie générale de la façon suivante

Mesures uniques sur 51 aulacodes mâles nés en captivité

- 23 animaux âgés de 4-5 mois (1 $084 \mathrm{~g}$ poids vif moyen) ;

- 10 animaux âgés de 7-8 mois (1 $706 \mathrm{~g}$ poids vif moyen) ;

- 8 animaux âgés de 9-10 mois (2 957 g poids vif moyen) ;

- 10 animaux âgés de 11-12 mois (4 $596 \mathrm{~g}$ poids vif moyen).

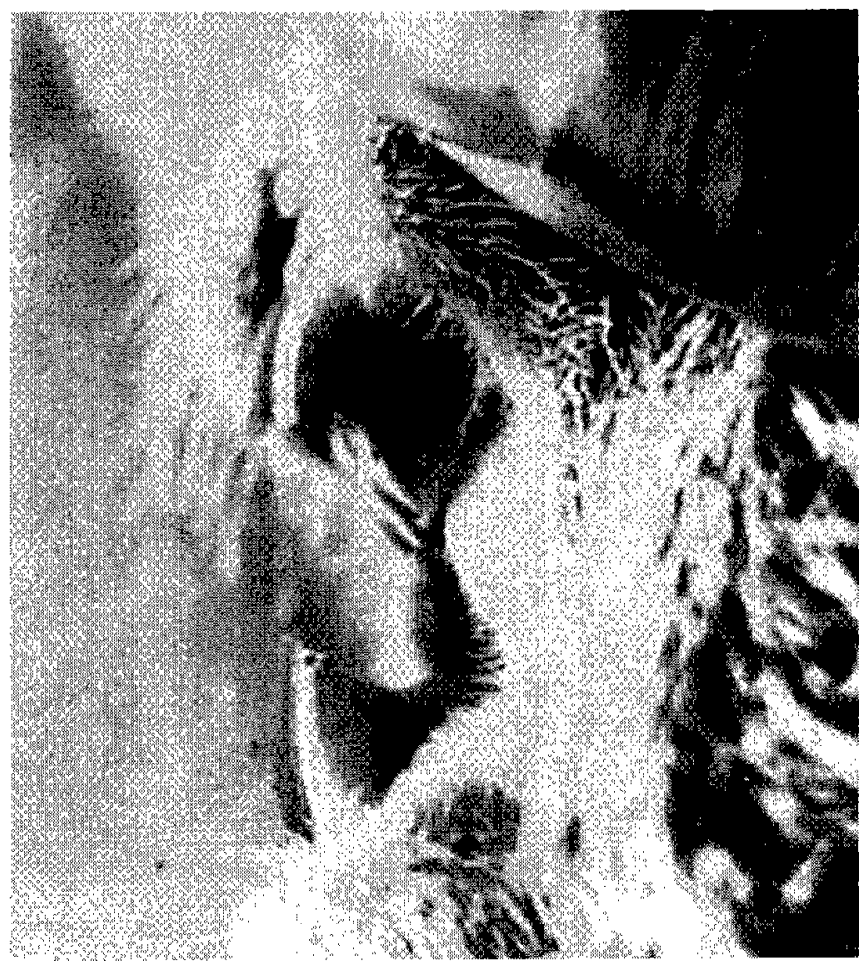

Photo 2 : Mauvaise usure du repli interne des incisives supérieures et apparition d'une fine lame.

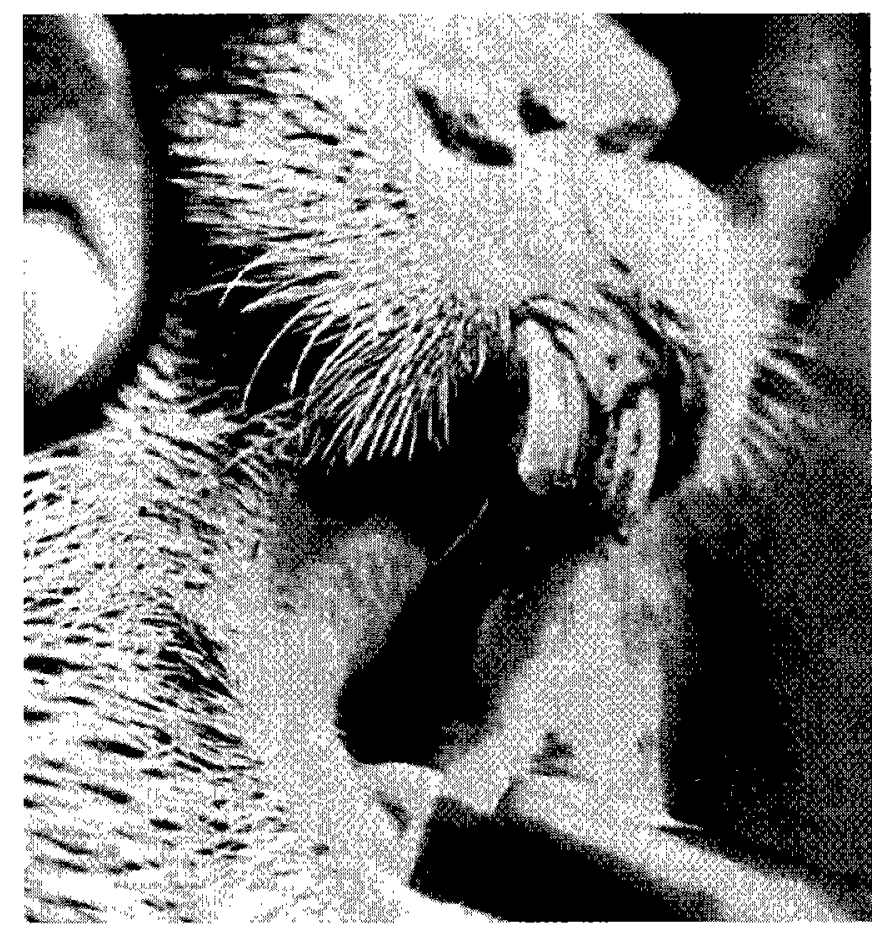

Photo 3 : Mauvaise usure du repli interne des incisives supérieures et formation d' une fine lame qui s'implantera dans le palais.

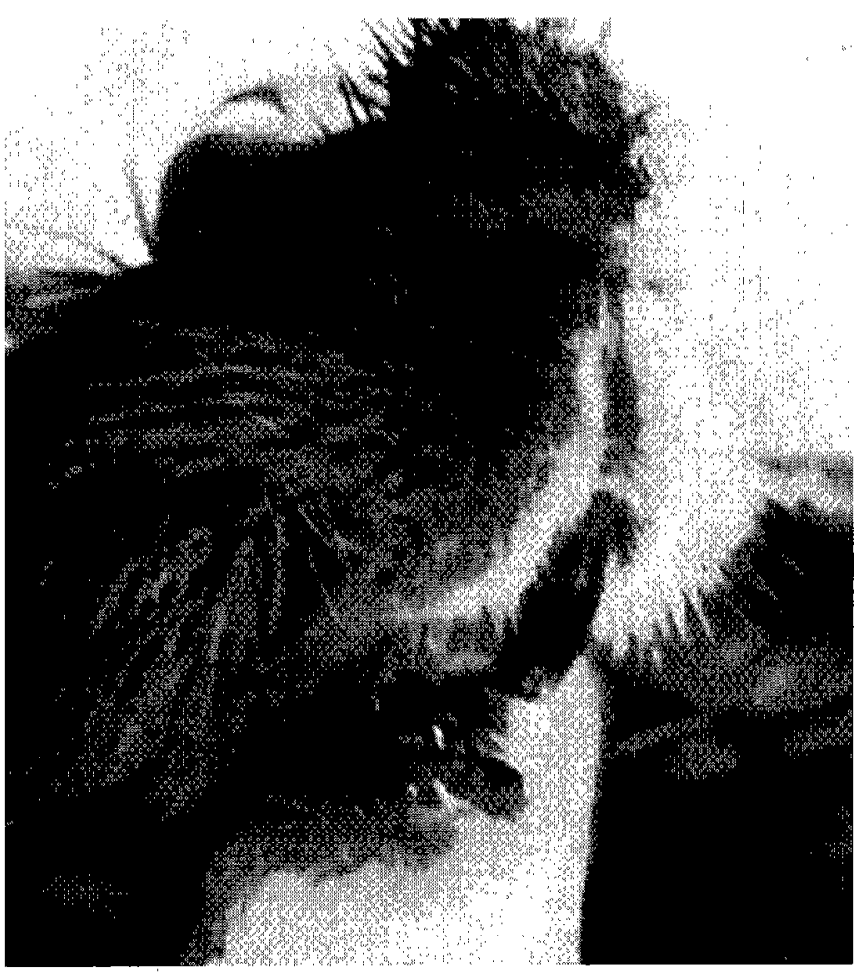

Photo $4:$ Formation d'une table d'usure au niveau des incisives infé reures. 
Au moment des expériences il n'y avait pas d'aulacodes mâles âgés de 6 mois. C'est ce qui explique le manque d'information dans cette classe d'âge. Les mesures prises sont les suivantes:

- poids vif ;

- longueur des incisives supérieures et inférieures ;

- largeur de la face labiale des incisives supérieures et inférieures ensemble.

Mesures répétées trois fois à intervalle régulier de temps

Sur 30 aulacodes répartis en 3 groupes de 10 animaux chacun :

- groupe 1 : âgés de 4-5 mois, mesures tous les 3 jours ;

- groupe 2 : âgés de 7-8 mois, mesures tous les 5 jours ;

- groupe 3 : âgés de 11-12 mois, mesures tous les 7 jours.

Les mesures prises portent sur:

- croissance des incisives supérieures ;

- usure des incisives supérieures.

Les incisives supérieures ont été marquées à 1-2 mm de la gencive à l'aide d'une petite scie. Les mesures linéaires ont été faites à l'aide d'un pied à coulisse. Chaque fois que la marque était à $3 \mathrm{~mm}$ environ de l'extrémité libre de l'incisive, une nouvelle marque était faite toujours à 1-2 $\mathrm{mm}$ de la gencive. Les paramètres suivants ont été étudiés :

- croissance $=$ distance de la marque à la gencive, au départ et à une date $\mathrm{t}$ (en $\mathrm{mm}$ ) ;

- usure = distance de la marque à l'extrémité libre, au départ et à une date $\mathrm{t}$ (en $\mathrm{mm}$ ) :

- surcroît = croissance - usure.

Les données ont été analysées statistiquement.

\section{Résultats et discussion}

La figure 2 schématise respectivement les mesures des incisives supérieures et inférieures dans chaque classe d'âge. Les différences observées au niveau de la longueur des incisives sont significatives $(p \leq 0,05)$. D'une façon générale, les incisives inférieures de l'aulacode sont plus longues que les supérieures et les incisives croissent en fonction de l'âge. Les largeurs des incisives supérieures et inférieures sont presque identiques.

La figure 3 montre la croissance et l'usure quotidiennes des incisives supérieures. Le surcroît apparaît comme la différence entre croissance et usure. Le surcroît augmente avec l'âge mais les différences observées au niveau de l'usure et de la croissance des incisives supérieures entre les classes d'âge ne sont pas toutes statistiquement significatives. En effet, les aulacodes âgés de 11-12 mois ont une croissance moyenne des incisives supérieures de $0,055 \mathrm{~mm} /$ jour contre $0,047 \mathrm{~mm} /$ jour pour la classe $7-8$



Figure 2 : Dimensions moyennes des incisives supérieures et inférieures en fonction de l'âge.



Figure 3 : Croissance et usure des incisives supérieures par jour (valeurs moyennes du moindre carré).

mois et $0,039 \mathrm{~mm} / \mathrm{jour}$ pour la classe $4-5$ mois. L'usure moyenne des incisives chez les animaux de 11-12 mois est supérieure de $0,002 \mathrm{~mm} / \mathrm{jour}$ à celle de la classe $7-8$ 


\section{Communication}

mois, et de $0,007 \mathrm{~mm} / \mathrm{jour}$ à celle de la classe $4-5$ mois (fig. 3).

Ces chiffres relatifs à la croissance des incisives concordent avec ceux observés chez certains lagomorphes et rongeurs adultes. En effet, chez des lapins adultes les incisives croissent de $11-12 \mathrm{~cm} / \mathrm{an}$ (5) et chez des rats adultes de 2 à $3 \mathrm{~mm} / \mathrm{semaine}(4)$. Par contre nos chiffres sont inférieurs à ceux enregistrés chez des jeunes rats dont les incisives croissent de $0,1 \mathrm{~mm} /$ jour (6). Le surcroît enregistré chez des rats adultes a été de $0,015 \mathrm{~mm} / \mathrm{jour}$ (7). Notons cependant que durant cette expérimentation, certains des problèmes dentaires décrits par ADJANOHOUN $(1,2)$ n'ont pas été rencontrés.

\section{Conclusion}

Avec un surcroît de pousse des incisives supérieures compris entre 0,006 et $0,015 \mathrm{~mm} / \mathrm{jour}$, aucun problème dentaire n'a été observé. Toutefois, comme le stress ralentit forcement l'usure des dents et conduit à la mort par inanition, d'autres travaux sont menés pour résoudre le problème de l'usure dentaire insuffisante.

\section{Remerciements}

Nous remercions Mr. L.T. YEWADAN et le personnel du Département de zootechnie du Projet bénino-allemand d'aulacodilculture qui nous ont permis de faire les mesures avec les aulacodes de leur cheptel, et nous ont aussi aidé durant la collecte des données expérimentales.
MENSAH (G.A.), BRÖNNEMANN (A.), STIER (C.H.), GALL (C.F.), Practical aspects of grass-cutter (Thryonomys swinderianus) breeding. V. Normal growth and wear of incisors. Revue Élev. Méd. vét. Pays trop. $1992,45(2): 175-178$

During the breeding of grass-cutters in captivity, problems resulting from incisors in bad conditions and insufficient wear were observed. In order to understand the origin of this phenomen incisor growth and wear were studied in grass-cutters aged between 4 and 12 months. Incisor growth was $0.039-0.055 \mathrm{~mm}$ per day and wear was $0.033-0.040 \mathrm{~mm}$ per day. Accordingly growth excess was $0.006-0.015 \mathrm{~mm}$ per day but did not cause any dental problem. Key words : Grass-cutter - Thryonomys swinderianus Breeding - Teeth - Wear - Benin.

\section{Bibliographie}

1. ADJANOHOUN (E.). L'usure anormale des incisives de l'aulacode : un problème majeur de l'élevage en captivité. Rapport technique inédit, PBAA/DEP/MDRAC/BENIN, 1986

2. ADJANOHOUN (E.). Bilan de l'état des incisives de tous les aulacodes entretenus au PBAA au 6 septembre 1986. Rapport technique inédit PBAA/DEF/MDRAC/BENIN, 1986

3. ALIDOU (A.K.). Connaissances actuelles sur la pathologie de l'aulacode Thryonomys swinderianus (Temminck 1827) en captivité étroite au Projet Bénino-Allemand d'Aulacodi-culture. Mém., Université Nationale du Bénin, 1987.

4.EISENMENGER (E.). T'ierärztliche Zahnheilkunde. 'T'hieme, 4-27, 1982.

5. HABERMEHL (K.H.). Die Altersbestimmung bei Versuchstieren. Berlin und Hamburg, Paul Parcy, 1980.

6. MOHIUDDIN (A.). Studies in modified growth in mammals. Part I Pakist.J.Sci., 1957, $9: 261-265$.

7. RAJA (Z.A.), FATANI (J.A.). Rate of growth of lower incisor teeth in the adult white rat. Acta Anat., 1977, $9: 84-85$. 